\title{
ACCROISSEMENT DE LA CONTRIBUTION DES SOURCES D'EROSION AUX RIVIERES ET PLANS D'EAU (1950-2010) : LE CAS DU LOUROUX (INDRE-ET-LOIRE, FRANCE)
}

Anthony FOUCHER ${ }^{(1)}$, Marion LE GALL ${ }^{(2)}$, Sébastien SALVADOR-BLANES ${ }^{(1)}$, Olivier EVRARD $^{(2)}$, Olivier CERDAN ${ }^{(3)}$, John-Patrick LACEBY ${ }^{(2)}$, Rosalie VANDROMME ${ }^{(3)}$, Irène LEFEVRE $^{(2)}$, Louis MANIERE ${ }^{(1)}$, Thomas GRANGEON ${ }^{(1,3)}$, Jean-Paul BAKYONO ${ }^{(1)}$, Marc $\operatorname{DESMET}^{(1)}$

(1) Laboratoire GéoHydrosystèmes Continentaux (GéHCO), E.A 6293, Université F. Rabelais de Tours, Faculté des Sciences et Techniques, Parc de Grandmont, 37200 Tours, France - e-mail: anthony.foucher@outlook.com

${ }^{(2)}$ Laboratoire des Sciences du Climat et de l'Environnement (CEA/CNRS/UVSQ), Université Paris-Saclay, Gif-sur-Yvette, France

${ }^{(3)}$ Département Risques et Prévention, Bureau de Recherches Géologiques et Minières (BRGM), 3 avenue Claude Guillemin, 45060 Orléans, France

\begin{abstract}
L'érosion des sols est reconnue comme étant l'un des principaux processus à l'origine de la dégradation physicochimique des masses d'eau. Les phénomènes érosifs, largement décrits en contexte morphogène, restent pourtant peu étudiés en contexte de plaine.

Pour combler ce manque, une étude pluridisciplinaire a été menée à l'échelle d'un petit bassin versant $\left(25 \mathrm{~km}^{2}\right)$ de plaine agricole drainée pour reconstruire l'évolution des taux et des sources d'érosion, depuis les zones d'accumulation de matière jusqu'aux zones sources. Cette étude vise ainsi à comprendre l'impact des aménagements intervenus dans le bassin après 1950 (remembrements, création de fossés, drainage) sur la dynamique sédimentaire actuelle et passée.

À cette fin, des carottages ont été réalisés dans un étang médiéval (870AD) localisé à l'exutoire du bassin versant du Louroux (France). Les carottes ont été datées et ont permis de reconstruire les variations temporelles du flux sédimentaire (de 1954 à 2013), mais aussi de les associer avec les changements d'usage des sols. Par ailleurs, les sources de sédiments ont été identifiées à partir de mesures de radionucléides (césium 137 et rapport béryllium 7/plomb 210 en excès). Ces résultats vont permettre de guider la mise en place d'aménagements pour lutter contre le départ de matière sur les versants.
\end{abstract}

MOTS CLEFS : érosion, sédiment, colmatage, archive sédimentaire, traçage

\section{Increase of erosion source contributions to rivers and lakes (1950-2010): the case of the Louroux Pond (Central France)}

\begin{abstract}
Soil erosion is recognized as one of the main processes triggering the physical and chemical degradation of water bodies. Although erosive dynamics have been widely described in morphogenic areas, much less is known about this process in lowland agricultural catchments.

To address this issue, a multidisciplinary study was carried out at the scale of a small agricultural lowland catchment (Louroux catchment, France, $25 \mathrm{~km}^{2}$ ) to reconstruct the evolution of the soil erosion rates and sources, from the hillslopes to the deposition areas in the water bodies. This study's goal is specifically to understand the impact of changes in land use and agricultural practices after 1950 (field re-design, ditch creation, drainage) on sediment dynamics

For this purpose, sediment cores were collected in a medieval pond (870AD) located at the outlet of the catchment. These cores have been dated and used to reconstruct the temporal variations in sediment fluxes over time (from 1954 to 2013). In addition, sediment sources were identified using fallout radionuclide measurements (cesium-137 and beryllium7 / excess lead-210 ratios). These results will contribute to the implementation of effective management measures to reduce the transfer of sediments from the hillslopes to the hydrosystems.
\end{abstract}

KEY WORDS: erosion, sediment, siltation, lake deposits, sediment fingerprinting 


\section{INTRODUCTION}

En quelques décennies, les paysages agricoles français et européens ont subi des changements rapides et radicaux d'occupation des sols. Ces bouleversements ont favorisé l'implantation et le développement de nouveaux systèmes culturaux, de plus en plus productivistes. Les paysages ruraux ont été remodelés et les parcelles remembrées [Chartin et al., 2011; Chartin et al., 2013], entrainant une suppression des élément linéaires et une ouverture du paysage.

Suite à ces aménagements, les phénomènes érosifs se sont accélérés et intensifiés [Wilkinson, 2005] menaçant la durabilité des ressources en sol et la viabilité des écosystèmes naturels [Commission of the European Communities, 2006] . Au-delà des conséquences locales sur la dégradation des potentialités agronomiques des sols [Bakker et al., 2008; Boardman et Poesen, 2006], l'accroissement de ces phénomènes érosifs a accentué l'apport de sédiments aux rivières. Ces sédiments issus des versants sont susceptibles d'être transférés vers les environnements aquatiques. Au Royaume-Uni, on estime que $70 \%$ des sédiments circulant dans les cours d'eau proviennent des terres agricoles [Rickson, 2014] .

Dans les zones agricoles, anthropisées, où les aménagements sur les versants sont largement développés (réseaux de fossés et de drainage), ces transferts de matière depuis les versants sont accélérés par une connectivité optimale de la source au cours d'eau [López-Vicente et al., 2013]. Cette notion de connectivité est majeure et de plus en plus régulièrement prise en compte dans les études sédimentaires [Gay et al., 2014]. Elle traduit le fait que, si la vulnérabilité à l'érosion sur une parcelle donnée peut-être élevée, elle ne transfèrera pas de particules au cours d'eau situé à l'aval si elle n'est pas directement reliée à celui-ci. À l'inverse, les parcelles à faible risque d'érosion peuvent présenter un risque environnemental fort si la connectivité au cours d'eau est forte, ne fût-ce qu'une partie de l'année [Rickson, 2014] .

Une fois transférés depuis les zones d'érosion vers les masses d'eau par une connexion plus ou moins rapide, ces sédiments vont participer au comblement et à l'envasement des lacs, des étangs, mais également à celui de certaines sections des cours d'eau [Dearing, 1991].

Au-delà des conséquences financières liées aux coûts de gestion de ces comblements des retenues hydroélectriques ou du colmatage des retenues collinaires [Ben Slimane et al., 2013], ces transferts massifs de matériaux provoquent une multitude de conséquences négatives pour les habitats aquatiques et pour l'accès à une ressource en eau de qualité (réduction de la pénétration de la lumière [Owens et al., 2005], destruction des habitats et des frayères de la faune benthique [Massa, 2000], ou encore détérioration des branchies des poissons [Waters, 1995]). Lors de ce transfert sédimentaire, les matières en suspension participent également à la modification de la composition chimique du milieu aquatique et elles constituent une source importante de pollution diffuse pour les eaux superficielles [Davis et Fox, 2009; Evrard et al., 2016; Hodgkinson et Withers, 1996]. Ces sédiments se caractérisent par un pouvoir cohésif et une capacité de sorption élevé vis-à-vis des nutriments, des métaux lourds, des pesticides et autres contaminants organiques [Shear et Watson, 1977; Vidon et Cuadra, 2011]. On retrouvera donc - au niveau de ces sédiments qui transitent et s'accumulent dans les masses d'eau - des teneurs importantes en polluants liés aux intrants utilisés en agriculture [Sabatier et al., 2014].

Les changements passés d'usage des sols ont ainsi impacté et impactent encore aujourd'hui tous les compartiments des hydrosystèmes et, ce, à l'échelle globale. Ces enjeux environnementaux sont d'une telle importance que la Commission européenne a fait de la lutte contre l'érosion des sols l'une des priorités de sa politique environnementale [Commission of the European Communities, 2006].

Les références scientifiques soulevant les effets néfastes de cette anthropisation brutale des milieux agricoles commencent à se multiplier à partir des années 1980 [Dearing et al., 1987; Lambert et Walling, 1988; Walling, 1983]. Néanmoins, la quantification des conséquences environnementales de ces aménagements reste complexe compte-tenu de l'absence de données historiques sur la qualité physico-chimique des cours d'eau. Pour pallier ce manque de données historiques, la collecte et l'étude d'archives sédimentaires offre une opportunité unique de réaliser ces reconstructions. Des travaux de recherche similaires ont déjà été entrepris dans les contextes morphogènes mais, à notre connaissance, peu d'études sont disponibles en contexte de plaines agricoles fortement anthropisées.

Au-delà de cette reconstruction temporelle des flux sédimentaires, l'identification de l'origine spatiale des sources de sédiments et leur évolution temporelle saisonnière constituent un enjeu majeur pour envisager la mise en place de dispositifs efficaces permettant de lutter contre l'érosion en amont des masses d'eau.

Les travaux présentés dans cet article sont le fruit d'un travail collaboratif visant à comprendre et à quantifier l'impact des aménagements passés d'un bassin agricole sur la déstabilisation de la cascade sédimentaire, en remontant depuis les zones d'accumulation, où des archives sédimentaires ont été prélevées, 
jusqu'à l'identification des zones sources à l'amont qui participent au comblement des masses d'eau. L'étude présentée ici a été réalisée dans un domaine de plaine agricole drainée d'Europe de l'Ouest, dans une région et un contexte très peu décrits dans la littérature [Vanmaercke et al., 2011].

Nous présenterons ici (1) la reconstruction et la quantification des flux sédimentaires de l'étang médiéval du Louroux (Indre-et-Loire) [Foucher et al., 2014], (2) l'identification des sources des sédiments qui comblent l'étang à l'aide du radio-isotope artificiel césium $137\left({ }^{137} \mathrm{Cs}\right)$ - [Foucher et al., 2015; Le Gall et al., 2016], (3) l'estimation du temps de transfert des particules depuis les zones sources jusqu'à l'étang du Louroux, grâce aux concentrations de deux radionucléides naturels, le béryllium $7\left({ }^{7} \mathrm{Be}\right)$ et le plomb 210 en excès $\left({ }^{210} \mathrm{~Pb}_{\mathrm{xs}}\right)$ [Le Gall et al., 2017].

\section{SITE D'ETUDE ET METHODES}

\section{II.1 Présentation du site d'étude}

Le bassin versant du Louroux $\left(25 \mathrm{~km}^{2}\right)$ est situé dans la partie sud-ouest du bassin parisien, dans le bassin hydrographique de la Loire (Fig. 1). Il est caractérisé par une topographie très plane (pente moyenne de $0,44 \%$ ) avec une altitude comprise entre 94 et $128 \mathrm{~m}$. 78\% de la surface du bassin sont occupés par des terres arables, $18 \%$ par des prairies et $4 \%$ par des forêts [Corine Land Cover, 2002] . Le climat sur ce secteur est de type océanique tempéré avec des précipitations moyennes annuelles de 684mm (entre 1971 et 2000).
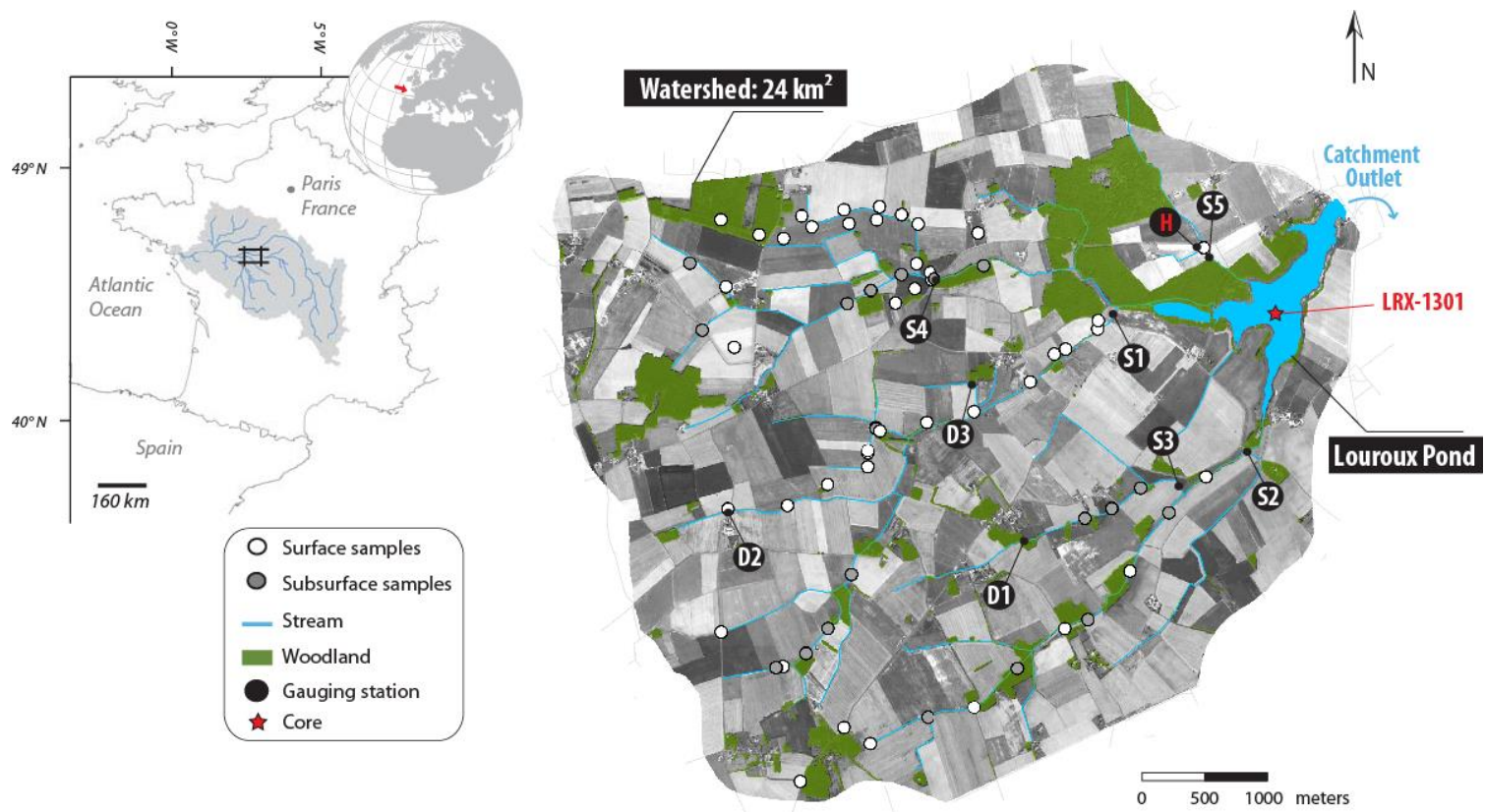

Fig. 1: Localisation du site d'étude du bassin versant du Louroux. Localisation des échantillons de sources potentielles de sédiments (surface des sols, subsurface des sols), des stations de mesures sur le réseau hydrographique $(\mathrm{S}=$ station sur cours d'eau, $\mathrm{D}$ = station sur collecteur de drain) et du site du carottage dans l'étang.

Depuis les années 1950, le bassin versant du Louroux, comme la plupart des bassins agricoles d'Europe de l'Ouest, a subi d'importantes modifications de l'occupation des sols et un changement radical des pratiques agricoles. Deux campagnes de remembrements ont été mises en place (1954 et 1992).

À partir de 1944, des cours d'eau ont été creés et recalibrés. La longueur du réseau hydrographique est ainsi passée de $10 \mathrm{~km}$ avant 1944 à près de $45 \mathrm{~km}$ en 2015.

Pour évacuer l'eau des sols hydromorphes, de vastes réseaux de drainage agricole ont été installés (plus de 210 collecteurs de drains sont actuellement présents). Ce bassin est ainsi passé, en quelques décennies, d'une zone hydromorphe occupée majoritairement par des prairies destinées à l'élevage à une zone de grandes cultures à dominante céréalière.

L'étang du Louroux (52 ha), situé à l'exutoire du bassin versant constitue l'une des plus grandes et des plus anciennes masses d'eau (870AD) de la région. Elle reçoit les eaux de ruissellement et les sédiments en provenance des cinq principaux tributaires qui drainent le bassin versant. Cette masse d'eau qui fonctionne par débordement a potentiellement enregistré dans ses sédiments l'ensemble des modifications qui sont intervenues dans le bassin amont depuis les années 1950. Depuis 2013, les principaux tributaires de l'étang du 
Louroux sont équipés de stations permettant de mesurer le flux de sédiments transitant dans les cours d'eau et de collecter ponctuellement des sédiments (les stations sont ainsi équipées d'un préleveur automatique, d'une sonde de turbidité et d'une sonde de niveau d'eau) - [Foucher, 2015].

\section{II.2 Méthodes}

\section{II.2.1 Caractérisation et quantification des stocks sédimentaires}

Une approche limnogéologique a été menée sur l'ensemble de l'étang du Louroux pour caractériser et quantifier le volume de sédiments accumulés, ainsi que pour reconstruire l'évolution temporelle des apports terrigènes [Foucher, 2015].

D'un point de vue technique, 7,5 km de profils sismiques à haute résolution ont été acquis lors d'une campagne de réflexion sismique. La compilation de ces données a permis d'identifier les zones les plus représentatives de l'étang pour collecter des carottes sédimentaires. Trois carottes ont été collectées en 2013.

La carotte-maître (LRX13-01), prélevée au centre de l'étang (Fig. 1), a été datée à l'aide de radionucléides $\left({ }^{137} \mathrm{Cs}\right.$ et $\left.{ }^{210} \mathrm{~Pb}_{\mathrm{xs}}\right)$ et de marqueurs stratigraphiques connus par ailleurs (périodes de vidanges de l'étang) [Foucher et al., 2014]. À partir des mesures de densités (mesurées tous les $\mathrm{cm}$ ) et des datations, la courbe de Mass Accumulation Rate (MAR) a été calculée pour estimer l'évolution des apports de sédiments à l'étang au cours du temps [Van Metre et al., 2004]. Pour reconstituer la dynamique du flux terrigène provenant du bassin versant et s'affranchir de la production autochtone dans l'étang, des analyses quantitatives des palynofaciès ont été effectuées au sein des principales unités de la carotte-maître, selon la méthode proposée par Simonneau et al. [2013]. Ces différentes mesures effectuées sur les trois carottes ont permis de calibrer le signal acoustique acquis lors des mesures sismiques. Ce signal calibré a ensuite été utilisé pour estimer le volume de matériaux accumulé au cours des 10 dernières années dans l'étang (période 2003-2013).

\section{II.2.2 Identification de la source et quantification du temps de transfert des sédiments}

\section{Source spatiale}

Le ${ }^{137} \mathrm{Cs}$, qui est principalement retombé au sol lors des essais thermonucléaires atmosphériques des années 1960, permet de discriminer les particules de sol qui proviennent de la surface (marquée en ${ }^{137} \mathrm{Cs}$ ) ou, au contraire, de la subsurface (dépourvue de ${ }^{137} \mathrm{Cs}$ ). La mesure de ce radio-isotope tant dans les sources potentielles que dans les sédiments permet ainsi de définir la proportion de particules transitant dans les cours d'eau ou accumulées dans les plans d'eau qui provient de l'érosion de la surface des sols ou, au contraire, des berges et des ravines éventuelles [e.g Olley et al., 1993; Olley et al., 2013; Caitcheon et al., 2012]. Dans le contexte de plaine agricole drainée qui nous intéresse ici, des résultats antérieurs ont montré que les particules transitant dans les drains étaient marquées en ${ }^{137} \mathrm{Cs}$ et provenaient donc de la surface des sols cultivés [Foucher et al., 2014].

Pour conduire cette étude de traçage sédimentaire, des mesures de ${ }^{137} \mathrm{Cs}$ ont été réalisées sur les principales sources identifiées sur les versants ( sols $=34$, berges $n=15$ ) ainsi qu'au niveau des collecteurs de drains instrumentés ( $n=5)$ - (Fig. 1).

En parallèle, des matières en suspension ont été prélevées dans les principaux affluents de l'étang $(\mathrm{n}=23)$ afin de caractériser les propriétés de la matière qui y transite durant différents types de conditions hydrologiques (voir la localisation des stations de mesures et de prélèvements à la Fig. 1). De plus, des mesures granulométriques ont été réalisées sur les échantillons de sources et de sédiments (Malvern Mastersizer 3000). Ces mesures ont permis d'appliquer un facteur de correction granulométrique pour rendre comparables les teneurs en ${ }^{137} \mathrm{Cs}$ obtenues dans les sources potentielles, présentant une granulométrie plus grossière, et les sédiments plus fins transitant dans les cours d'eau [Collins et al., 1996; Foucher et al., 2015].

Les échantillons corrigés de l'effet granulométrique ont ensuite été utilisés pour la description statistique des données. Les distributions de l'activité de ces échantillons ont été modélisées suivant un échantillonnage hypercube latin, basé sur la médiane et l'écart-type de la distribution de chacune des sources. Ce modèle a déjà été testé avec succès lors de précédentes études [Laceby et Olley, 2015]. Il a été simulé 2500 fois afin d'optimiser la discrimination entre les sources de surface et celles de subsurface pour les échantillons de sédiments. L'ensemble de la méthode est décrit dans Foucher et al. [2015].

\section{Temps de transfert}


Le ${ }^{7} \mathrm{Be}$ et le ${ }^{210} \mathrm{~Pb}_{\mathrm{xs}}$, (présentant une demi-vie respective de 53 jours et de 22 ans) ont été utilisés pour contraindre la dynamique temporelle des sédiments et quantifier la proportion de sédiments récemment érodés ou, au contraire, remis en suspension après avoir été stockés dans le réseau hydrographique, ainsi que leur temps de résidence au sein de l'hydrosystème [Bonniwell et al., 1999; Evrard et al., 2010; Gourdin et al., 2014; Matisoff et al., 2005]. La méthode consiste à comparer les rapports entre le ${ }^{7} \mathrm{Be}$ et le ${ }^{210} \mathrm{~Pb}_{\mathrm{xs}}$ du signal d'entrée (qui traduit les apports récents de ${ }^{7} \mathrm{Be}$ par les retombées atmosphériques) à ceux qui sont mesurés au niveau des sédiments collectés dans les cours d'eau (Fig. 1). Les particules présentant un rapport ${ }^{7} \mathrm{Be} /{ }^{210} \mathrm{~Pb}$ xs proche de celui du signal entrant (donc élevé), correspondent à des particules marquées par le ${ }^{7} \mathrm{Be}$ de la pluie et, donc, érodées récemment. Au contraire, des particules caractérisées par un rapport ${ }^{7} \mathrm{Be}^{210} \mathrm{~Pb}_{\mathrm{xs}}$ faible (proche de 0) indiquent qu'elles n'ont pas été récemment marquées par le ${ }^{7} \mathrm{Be}$ de la pluie, et qu'elles sont donc plus anciennes.

Trois crues ont été échantillonnées entre 2013 et 2014, permettant de collecter 14 échantillons de matières en suspension au niveau des cinq stations de mesure situées sur les principaux affluents de l'étang ainsi qu'en sortie de collecteurs de drains $(n=4)$ - (Fig. 1). En 2016, deux crues supplémentaires ont été échantillonnées $(\mathrm{n}=19)$. Le signal d'entrée de la pluie a été collecté pour chacun de ces épisodes pluvieux à proximité de la station S5 (symbole H sur la Fig.1).

\section{RÉSULTATS ET DISCUSSIONS}

\section{III.1 Reconstruction de la dynamique sédimentaire}

La carotte prélevée au centre de l'étang a pu être datée par l'identification des pics de ${ }^{137} \mathrm{Cs}$ et la décroissance $\mathrm{du}{ }^{210} \mathrm{~Pb}_{\mathrm{xs}}$ avec la profondeur. La présence de ${ }^{137} \mathrm{Cs}$ à la base de la carotte nous indique que ces sédiments se sont déposés après 1954 (début des retombées thermonucléaires atmosphériques). La séquence correspond donc à $2,35 \mathrm{~m}$ de sédiments peu compactés et qui s'y sont accumulés en 59 ans (le calcul du modèle d'âge est détaillé dans Foucher et $a l$. [2014]).

La reconstitution des flux de matière - corrigés de la production autochtone - au cours du temps (Fig. 2) permet d'observer l'occurrence d'un pic maximal de production sédimentaire entre 1954 et 1964 (les données antérieures n'étant pas couvertes par la séquence). Cette production importante est synchrone avec la fin des grands aménagements sur le bassin du Louroux (remembrements, création de fossés, mise en place des drains). Ces grands travaux connexes, entrepris en 1944, et qui se sont poursuivis jusqu'à la fin des années 1970, ont favorisé une accélération de la production sédimentaire en passant d'un apport moyen de $40 \mathrm{t}^{\mathrm{an}} \mathrm{n}^{-1}$ avant les aménagements, entre l'an 1000 et 1945, à près de 12000-14000 t.an ${ }^{-1}$ durant la phase d'export maximal de matière enregistrée dans la séquence (1954-1960). Ces valeurs correspondent à une érosion moyenne sur les versants d'environ 500 à $600 \mathrm{t} . \mathrm{km}^{-2}$. $\mathrm{an}^{-1}$. Ces valeurs élevées comprennent le matériel exporté depuis les sols, les berges et les réseaux de drains.

Le flux sédimentaire décroît ensuite progressivement avec le ralentissement des travaux et la

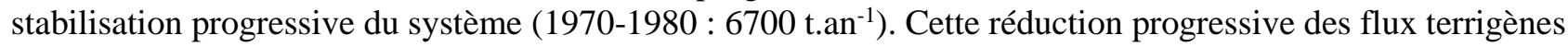
coïncide avec une réduction des aménagements, mais également avec l'eutrophisation croissante de l'étang qui se caractérise par une modification des communautés algales dans la masse d'eau. Le ralentissement de la production sédimentaire dans le bassin aurait donc été partiellement compensé par une augmentation de la production primaire dans l'étang.

Malgré ce retour vers une stabilité progressive, la masse d'eau reste très sensible aux aménagements du paysage réalisés en amont. Les derniers aménagements recensés sur le bassin au début des années 1990 (dernier remembrement) induisent une nouvelle et dernière augmentation limitée de la production sédimentaire qui passe de 2400 à 2700 t.an $^{-1}$. Le flux terrigène, qui tend à se stabiliser depuis les années 1980, reste néanmoins conséquent et il est près de 60 fois plus important que le flux enregistré avant les premiers aménagements des années 1950. 


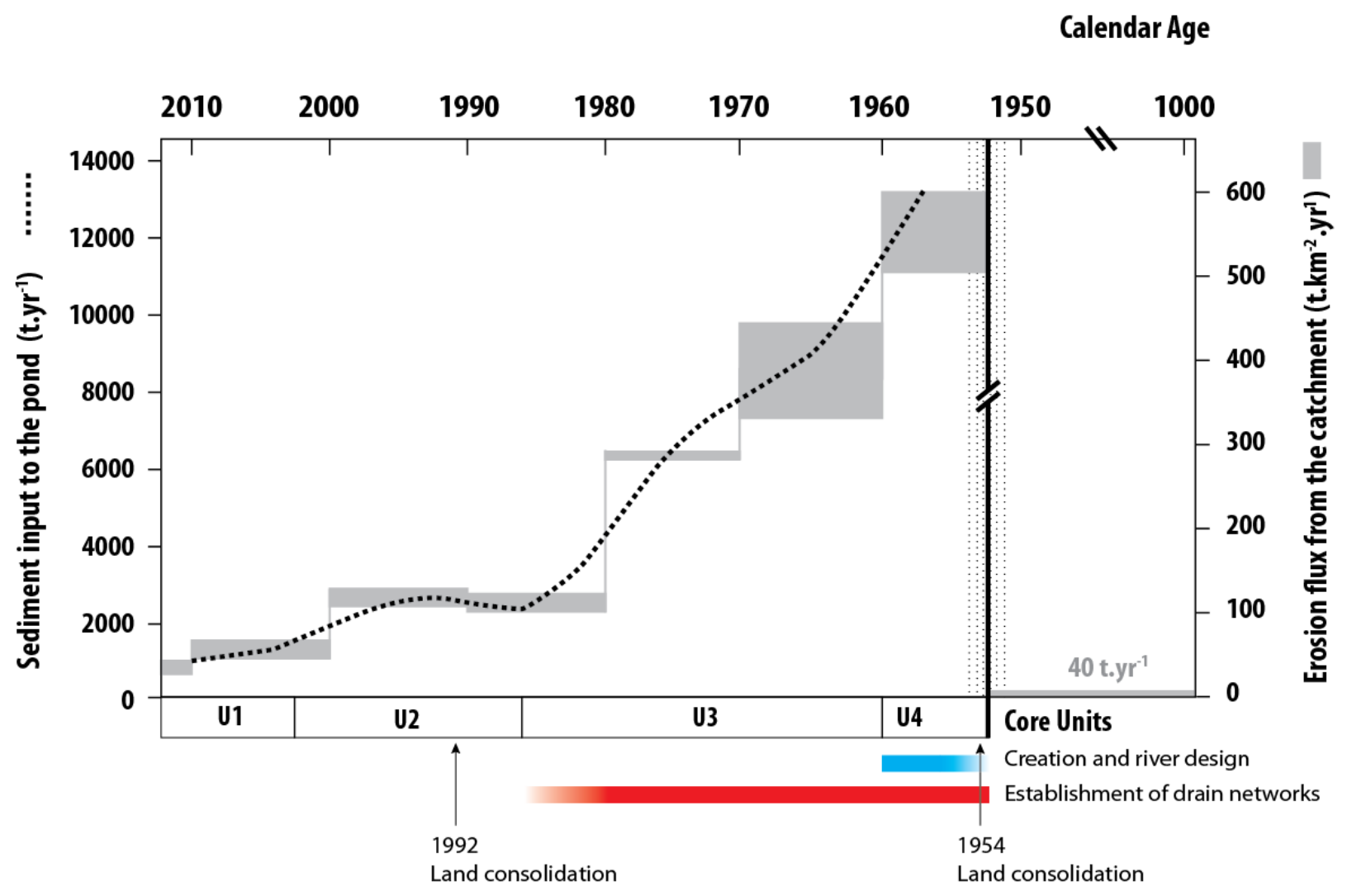

Fig. 2: Évolution de la dynamique sédimentaire (t.an $\left.{ }^{1}\right)$ et du flux érosif $\left(t \cdot \mathrm{km}^{-2} \cdot \mathrm{an}^{-1}\right)$ sur le basin versant du Louroux. Estimation effectuée à partir de la carotte-maître LRX-13-01 après datation et correction de la fraction autochtone modifié d'après Foucher et al. [2014].

Pour la période actuelle (2003-2013), la compilation des données sismiques et leur corrélation avec les caractéristiques des carottes sédimentaires ont permis d'estimer qu'en une dizaine d'années, près de 163 $000 \mathrm{~m}^{3}$ de matière (autochtone et allochtone) se sont accumulés dans cette masse d'eau de 52 ha, correspondant à une épaisseur moyenne de dépôts de l'ordre de $38 \mathrm{~cm}$. Sur ces $4890 \mathrm{t}^{-\mathrm{an}^{-1}}$ accumulées dans l'étang du Louroux, on peut estimer après analyse des palynofaciès qu'entre 2152 et 2445 t.an $^{-1}$ correspondent à de la matière terrigène en provenance des versants, le reste étant issu de la production primaire in situ.

Les tendances obtenues pour le bassin du Louroux sont semblables à celles qui ont été obtenues pour d'autres réservoirs en Europe et même ailleurs dans le monde [Dearing, 1991; Dearing et Jones, 2003; Foster et Walling, 1994; Heathcote et al., 2013]. Pourtant, ces derniers sites d'étude étaient localisés dans des contextes topographiques et d'occupation des sols bien plus contrastés que le bassin du Louroux. Les flux reconstruits pour la période 2003-2013 (90 à $100 \mathrm{t} . \mathrm{km}^{-2} \cdot \mathrm{an}^{-1}$ ) dans le bassin du Louroux sont également comparables avec la médiane du flux estimé pour l'Europe par Vanmaercke et al. [2011] et qui atteint 92 t.km ${ }^{2} \cdot \mathrm{an}^{-1}$.

La dynamique enregistrée ces dernières années pose toutefois de nombreuses questions sur la durabilité du système sol et la préservation des hydrosystèmes dans un tel environnement. On peut estimer à travers cette étude que, si la dynamique sédimentaire actuelle se poursuit, l'étang du Louroux sera comblé entre 2065 et 2115. Il est donc nécessaire de mieux identifier l'origine spatiale des sources de sédiments et leurs périodes de contributions principales afin de proposer des moyens de lutte efficaces contre l'érosion permettant de limiter autant que possible les apports terrigènes du bassin versant.

\section{III.2 Origine spatiale des sédiments}

La mise en suspension sélective et le transfert préférentiel des particules les plus fines lors de l'érosion se traduit par un enrichissement en ${ }^{137} \mathrm{Cs}$ des sédiments exportés par rapport à leurs sources potentielles (diamètres médian des particules des sédiments $=6 \mu \mathrm{m}$; celui des sources $=32$ à $36 \mu \mathrm{m}$ ).

Pour s'affranchir de ce biais granulométrique, deux facteurs de correction ont été employés. Le premier d'entre eux, classiquement utilisé dans la littérature internationale ayant trait aux études de traçage sédimentaire, est basé sur l'utilisation de la surface spécifique des particules obtenue par des mesures granulométriques laser 
[Collins et al., 1996]. Le second facteur de correction constitue l'une des originalités de ce travail, car il a démontré le potentiel d'utilisation du thorium comme facteur de correction granulométrique [Foucher et al., 2015]. L'application de ces deux paramètres correctifs dans le bassin du Louroux a permis la comparaison des teneurs en ${ }^{137} \mathrm{Cs}$ dans les sources potentielles et dans les sédiments qui varient en effet, après correction granulométrique, dans la même gamme de valeurs (les résultats des corrections granulométriques sont détaillés dans Foucher et al. [2015].

À l'issue des simulations effectuées sur les 28 échantillons de sédiments, les résultats indiquent clairement la dominance des sources de surface dans les sédiments collectés durant les périodes de crues. Ce constat se répète quelle que soit la période d'échantillonnage au cours de la saison hydrologique (de décembre à avril). L'effet de la croissance de la végétation au cours du printemps ne semble pas modifier cette contribution en protégeant les sols de l'érosion : la contribution de la source de particules superficielles varie pendant les événements de crue entre 95 et 99\%, en fonction du facteur de correction utilisé (Fig. 3).

Cette dominance de la contribution des sources de surface est clairement moindre durant les périodes d'étiage où l'on assiste à un mélange quasiment égal entre les sources de surface et de subsurface, ces dernières représentant entre 51 et $60 \%$ du flux de matières en suspension au cours de ces périodes. La période d'étiage domine la majeure partie de l'année sur les petits cours d'eau du bassin du Louroux. Les résultats du traçage, obtenus au niveau du site de carottage pour la période 2003-2013, indiquent une nouvelle fois la dominance des sources de surface (97 à 99\%). 76 échantillons ont par ailleurs été prélevés dans l'étang du Louroux pour obtenir des résultats davantage représentatifs des apports à l'échelle de l'ensemble de la masse d'eau. La contribution moyenne de la source de subsurface y atteint $18 \pm 1 \%$ - [Le Gall et al., 2016]. Des résultats obtenus par une autre approche géomorphologique se focalisant sur l'érosion des berges restent, eux aussi, dans la même gamme, avec une contribution des berges de l'ordre de 27 à 30\% [Foucher et al., 2017; Vandromme et al., 2017].

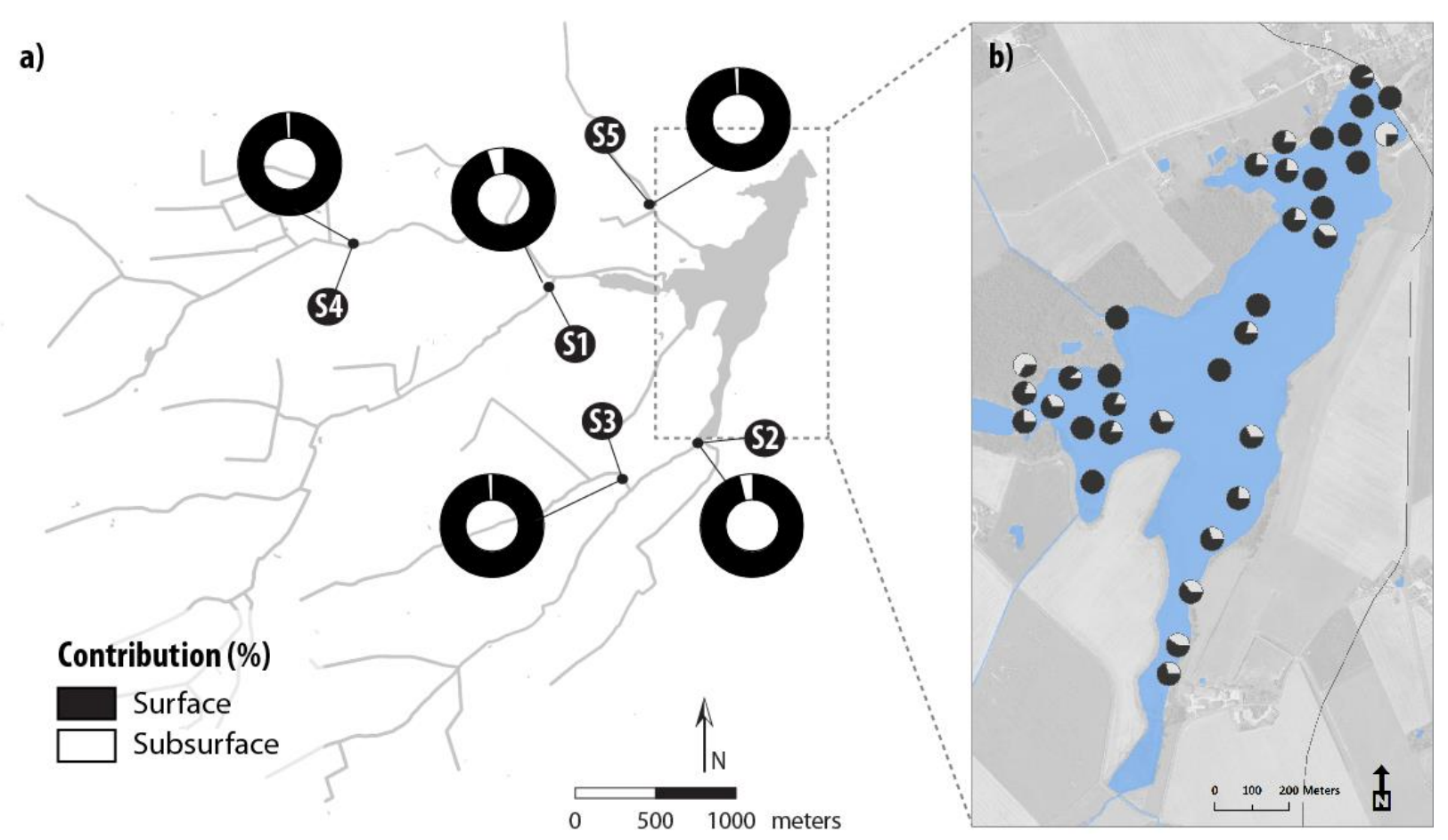

Fig. 3: Résultats du traçage des sources de sédiments dans les cours d'eau et l'étang du Louroux à l'aide du ${ }^{137}$ Cs. a) Contribution moyenne des sources de surface et de subsurface durant les crues de l'année hydrologique 2013/2014 d'après Foucher et $a l$. [2015] b) Contribution des sources dans le comblement de l'étang du Louroux d'après Le Gall et al. [2016].

Les mesures des teneurs en radionucléides dans les échantillons de sédiments prélevés aux exutoires des collecteurs de drains ont mis en évidence, quant à elles, des activités en ${ }^{137} \mathrm{Cs}$ élevées dans ces matières. Ces fortes valeurs confirment, comme cela a été suggéré dans de précédentes études [Sogon, 1999; Walling et al., 2002], une migration préférentielle de particules de surface marquées en césium au sein des profils de sols, au droit des drains. Ces résultats montrent donc que les sédiments provenant de la surface des sols peuvent transiter par différentes voies au sein du bassin : les dérayures et les figures d'érosion à la surface des sols ou 
le réseau de drainage. Le ruissellement des sols et la concentration des écoulements au sein des dérayures représentent ainsi les principaux pourvoyeurs de particules aux cours d'eau pendant les épisodes pluvieux (de décembre à janvier). Ensuite (au milieu /à la fin de l'hiver), le transfert de l'eau via les réseaux de drainage domine l'apport de particules au réseau hydrographique.

\section{III.3 Dynamique temporelle des sédiments}

Le rapport ${ }^{7} \mathrm{Be} /{ }^{210} \mathrm{~Pb}_{\mathrm{xs}}$ a été utilisé pour estimer la fraction récemment érodée et le temps de résidence des MES dans le bassin versant lors des différentes crues échantillonnées. Ces paramètres ont pu être estimés lors de chacune de ces crues et pour chacune des stations. Les résultats indiquent que la contribution moyenne aux MES des particules récemment érodées augmente d'une crue à l'autre lors d'une même saison, avec des contributions moyennes estimées entre $45 \pm 20 \%$ en décembre 2013 et $80 \pm 20 \%$ en février 2014 (Table 1). Lors des deux crues étudiées en janvier 2016, les contributions moyennes ont été respectivement estimées à $65 \pm 20 \%$ et $80 \pm 20 \%$, et ont donc également augmenté durant la saison hydrologique. L'évolution de la contribution des particules récemment érodées montre qu'en 2013-2014, lors des deux premières crues, les sédiments préalablement accumulés dans le chenal ou les drains ont été remis en suspension et progressivement exportés vers l'aval. A l'inverse, lors de la troisième crue, ce sont majoritairement les particules directement érodées de la surface des sols qui ont été exportées par les rivières vers l'étang situé à l'exutoire.

\begin{tabular}{lccc|ccc} 
& $1^{\text {st }}$ flood event $(30 / 12 / 2013)$ & $2^{\text {th }}$ flood event $(29 / 01 / 2014)$ & $3^{\text {th }}$ flood event (13/02/2014) \\
\hline Station & $\begin{array}{c}\text { \% of recently } \\
\text { eroded sediment }\end{array}$ & $\begin{array}{c}\text { Residence time } \\
\text { (days) }\end{array}$ & $\begin{array}{c}\text { \% of recently } \\
\text { eroded sediment }\end{array}$ & $\begin{array}{c}\text { Residence time } \\
\text { (days) }\end{array}$ & $\begin{array}{c}\text { \% of recently } \\
\text { eroded sediment }\end{array}$ & $\begin{array}{c}\text { Residence time } \\
\text { (days) }\end{array}$ \\
\hline Station 1 & $5 \pm 5$ & $220 \pm 60$ & $80 \pm 20$ & $20 \pm 1$ & $100 \pm 15$ & $1 \pm 3$ \\
Station 2 & $85 \pm 50$ & $10 \pm 40$ & $40 \pm 10$ & $65 \pm 1$ & $90 \pm 15$ & $4 \pm 3$ \\
Station 3 & $70 \pm 20$ & $25 \pm 15$ & $45 \pm 10$ & $60 \pm 2$ & $95 \pm 15$ & $4 \pm 3$ \\
Station 4 & $50 \pm 35$ & $50 \pm 40$ & $60 \pm 15$ & $40 \pm 2$ & $90 \pm 15$ & $10 \pm 5$ \\
Station 5 & $80 \pm 20$ & $20 \pm 15$ & $60 \pm 15$ & $40 \pm 2$ & $60 \pm 10$ & $40 \pm 3$ \\
Station d1 & $20 \pm 5$ & $135 \pm 10$ & $70 \pm 65$ & $25 \pm 50$ & & \\
Station d3 & $10 \pm 15$ & $165 \pm 90$ & $10 \pm 15$ & $100 \pm 55$ & &
\end{tabular}

Progressive exhaustion of sediment previously accumulated in the river channel

Table 1 : Estimations des temps de résidence (en jours) et des pourcentages de sédiments récemment érodés lors de crues de l'année hydrologique 2013-2014 pour chacune des stations de mesures (Fig. 1). D'après Le Gall [2016].

\section{CONCLUSIONS}

Les travaux menés sur le bassin versant du Louroux ont permis de reconstruire avec une haute résolution temporelle les conséquences environnementales de l'intensification des pratiques agricoles et des aménagements anthropiques dans le contexte particulier des plaines agricoles drainées. Les résultats ont montré la réponse synchrone de la dynamique sédimentaire/érosive avec la mise en place des aménagements sur le bassin versant, qui a subi une forte transition en passant d'un milieu peu connecté à l'atteinte d'une connectivité optimale (création de fossés, drains) en l'espace de quelques décennies à peine. La dynamique sédimentaire augmente très rapidement jusqu'à atteindre une production maximale entre les années 1954-1960 $\left(500\right.$ à $\left.600 \mathrm{t} \cdot \mathrm{km}^{-2} \cdot \mathrm{an}^{-1}\right)$ avant de décroître pour atteindre 90 à $100 \mathrm{t} \cdot \mathrm{km}^{-2} \cdot \mathrm{an}^{-1}$ entre 2003 et 2013. Malgré une chute et une stabilisation des apports depuis les années 1980, les flux actuels restent près de 60 fois plus importants que lors de la période précédant les aménagements. Ces apports induiront donc, si rien n'est fait d'ici là, un comblement total de l'étang du Louroux d'ici 50 à 100 ans.

Les résultats obtenus par le traçage sédimentaire ont montré l'importance de la contribution des sources de surface dans le comblement actuel de l'étang (82\%) et dans les transferts de MES durant les crues (95 à 99\%). Ces travaux ont également montré que les matières transitant dans les réseaux de drainage provenaient initialement de l'horizon de labour des sols. Les particules de la surface des sols sont donc mobilisées par deux voies de transfert, à savoir le ruissellement superficiel et les réseaux de drainage. À l'issue de cette étude, la contribution relative de chacune de ces voies de transfert n'a pas pu être établie, mais elle pourrait l'être à l'avenir en utilisant des modèles mathématiques ou en instrumentant des parcelles drainées. 
Enfin, les résultats obtenus par le traçage temporel ont montré une remobilisation et un export préférentiel des sédiments stockés dans le réseau hydrographique lors des premières crues de l'année hydrologique. On observe ensuite une contribution majoritaire des sources proches directement érodées de la surface des sols.

\section{REMERCIEMENTS}

Les travaux menés dans le cadre de cette étude ont été financés par l'Agence de l'Eau Loire Bretagne : des projets VERSEAU, TRACKSED et DRASTIC. Les auteurs souhaitent remercier Xavier Bourrain, Jean-Noël Gautier et Anne Colmar pour leurs conseils précieux et leur soutien lors des choix techniques dans le cadre des projets précités.

\section{REFERENCES}

Bakker, M.M., Govers, G., van Doorn, A., Quetier, F., Chouvardas, D., Rounsevell, M., 2008. The response of soil erosion and sediment export to land-use change in four areas of Europe: The importance of landscape pattern. Geomorphology 98, 213-226. doi:10.1016/j.geomorph.2006.12.027

Ben Slimane, A., Raclot, D., Evrard, O., Sanaa, M., Lefèvre, I., Ahmadi, M., Tounsi, M., Rumpel, C., Ben Mammou, A., Le Bissonnais, Y., 2013. Fingerprinting sediment sources in the outlet reservoir of a hilly cultivated catchment of Tunisia. . J. Soils Sediments 13, 801-815.

Boardman, J., Poesen, J., 2006. Soil Erosion in Europe. John Wiley \& Sons, Ltd, Chichester, UK. doi:10.1002/0470859202

Bonniwell, E.C., Matisoff, G., Whiting, P.J., 1999. Determining the times and distances of particle transit in a mountain stream using fallout radionuclides. Geomorphology 27, 75-92. doi:10.1016/S0169555X(98)00091-9

Caitcheon, G.G., Olley, J.M., Pantus, F., Hancock, G., Leslie, C., 2012. The dominant erosion processes supplying fine sediment to three major rivers in tropical Australia, the Daly (NT), Mitchell (Qld) and Flinders (Qld) Rivers. Geomorphology. doi:10.1016/j.geomorph.2012.02.001

Chartin, C., Bourennane, H., Salvador-Blanes, S., Hinschberger, F., Macaire, J.J., 2011. Classification and mapping of anthropogenic landforms on cultivated hillslopes using DEMs and soil thickness data: Example from the SW Parisian Basin, France. Geomorphology 135, 820.

Chartin, C., Evrard, O., Salvador-Blanes, S., Hinschberger, F., Van Oost, K., Lefèvre, I., Daroussin, J., Macaire, J.-J., 2013. Quantifying and modelling the impact of land consolidation and field borders on soil redistribution in agricultural landscapes (1954-2009). CATENA 110, 184-195. doi:10.1016/j.catena.2013.06.006

Collins, A.L., Walling, D.E., Leeks, G.J.L., 1996. Composite fingerprinting of the spatial source of fluvial suspended sediment : a case study of the Exe and Severn river basins, United Kingdom. Géomorphologie Reli. Process. Environ. 41-53.

CorineLandCover, 2002. EEA-ETC/TE. 2002. CORINE land cover update. I\&CLC2000 project. Technical guidelines, http://terrestrial.eionet.eu.int.

Davis, C.M., Fox, J.F., 2009. Sediment fingerprinting: review of the method and future improvements for allocating nonpoint source pollution. J. Environ. Eng. 135, 490-504.

Dearing, J.A., 1991. Lake sediment records of erosional processes. Hydrobiologia. doi:10.1007/BF00050938

Dearing, J.A., H\&\#xe5;kansson, H., Liedberg-J\&\#xf6;nsson, B., Persson, A., Skansj\&\#xf6;, S., Widholm, D., El-Daoushy, F., 1987. Lake Sediments Used to Quantify the Erosional Response to Land Use Change in Southern Sweden. Oikos 50, 60-78. doi:10.2307/3565402

Dearing, J.A., Jones, R.T., 2003. Coupling temporal and spatial dimensions of global sediment flux through lake and marine sediment records. Glob. Planet. Change 39, 147-168. doi:http://dx.doi.org/10.1016/S0921-8181(03)00022-5

Evrard, O., Laceby, J.P., Onda, Y., Wakiyama, Y., Jaegler, H., Lefèvre, I., 2016. Quantifying the dilution of the radiocesium contamination in Fukushima coastal river sediment (2011-2015). Sci. Rep. 6, 34828. doi: $10.1038 /$ srep34828

Evrard, O., Némery, J., Gratiot, N., Duvert, C., Ayrault, S., Lefèvre, I., Poulenard, J., Prat, C., Bonté, P., Esteves, M., 2010. Sediment dynamics during the rainy season in tropical highland catchments of central Mexico using fallout radionuclides. Geomorphology 124, 42-54. doi:10.1016/j.geomorph.2010.08.007

Foster, I.D.L., Walling, D.E., 1994. Using reservoir deposits to reconstruct changing sediment yields and sources in the catchment of the Old Mill Reservoir, South Devon, UK, Over the past 50 years. . Hydrol. Sci. 347-368.

Foucher, A., 2015. Reconstruction of sediment supply in intensively cultivated lowlands : sources, pathways 
and sediment storage in the Louroux catchment (France). Thèses. Université de Tours. doi:10.13140/RG.2.2.24718.56643

Foucher, A., Patrick Laceby, J., Salvador-Blanes, S., Evrard, O., Le Gall, M., Lefèvre, I., Cerdan, O., Rajkumar, V., Desmet, M., 2015. Quantifying the dominant sources of sediment in a drained lowland agricultural catchment: The application of a thorium-based particle size correction in sediment fingerprinting. Geomorphology. 250. doi:10.1016/j.geomorph.2015.09.007

Foucher, A., Salvador-Blanes, S., Evrard, O., Simonneau, A., Chapron, E., Courp, T., Cerdan, O., Lefèvre, I., Adriaensen, H., Lecompte, F., Desmet, M., 2014. Increase in soil erosion after agricultural intensification: Evidence from a lowland basin in France. Anthropocene 7, 30-41. doi:10.1016/j.ancene.2015.02.001

Foucher, A., Sébastien, S.-B., Rosalie, V., Olivier, C., Marc, D., 2017. Quantification of bank erosion in a drained agricultural lowland catchment. Hydrol. Process. 31. doi:10.1002/hyp.11117

Gay, A., Cerdan, O., Delmas, M., Desmet, M., 2014. Variability of suspended sediment yields within the Loire river basin (France). J. Hydrol. 519, Part, 1225-1237.

Gourdin, E., Evrard, O., Huon, S., Reyss, J.-L., Ribolzi, O., Bariac, T., Sengtaheuanghoung, O., Ayrault, S., 2014. Spatial and temporal variability of $7 \mathrm{Be}$ and $210 \mathrm{~Pb}$ wet deposition during four successive monsoon storms in a catchment of northern Laos. J. Environ. Radioact. 136, 195-205. doi:10.1016/j.jenvrad.2014.06.008

Heathcote, A.J., Filstrup, C.T., Downing, J.A., 2013. Watershed Sediment Losses to Lakes Accelerating Despite Agricultural Soil Conservation Efforts. PLoS One 8, e53554. doi:10.1371/journal.pone.0053554

Hodgkinson, R.A., Withers, P.J.A., 1996. Non-point source phosphorus loss from small agricultural catchments. Diffus. Pollut. Agric. Scottish Agric. Coll. 120-126.

Laceby, J.P., Olley, J., 2015. An examination of geochemical modelling approaches to tracing sediment sources incorporating distribution mixing and elemental correlations. Hydrol. Process. 29, 1669-1685. doi:10.1002/hyp.10287

Lambert, C.P., Walling, D.E., 1988. Measurement of channel storage of suspended sediment in a gravel-bed river. CATENA 15, 65-80.

Le Gall, M., 2016. Traçage des sources de sédiments à l'amont des hydrosystèmes agricoles : apport de la géochimie élémentaire, du rapport $\mathrm{Sr} / \mathrm{Sr}$ et des radionucléides. Thèse. Université Paris-Saclay.

Le Gall, M., Evrard, O., Foucher, A., Laceby, J.P., Salvador-Blanes, S., Manière, L., Lefèvre, I., Cerdan, O., Ayrault, S., 2017. Investigating the temporal dynamics of suspended sediment during flood events with 7Be and 210Pbxs measurements in a drained lowland catchment. Sci. Rep. 7, 42099.

Le Gall, M., Evrard, O., Foucher, A., Laceby, J.P., Salvador-Blanes, S., Thil, F., Dapoigny, A., Lefèvre, I., Cerdan, O., Ayrault, S., 2016. Quantifying sediment sources in a lowland agricultural catchment pond using $137 \mathrm{Cs}$ activities and radiogenic $87 \mathrm{Sr} / 86 \mathrm{Sr}$ ratios. Sci. Total Environ. 566-567, 968-980. doi:10.1016/j.scitotenv.2016.05.093

López-Vicente, M., Poesen, J., Navas, A., Gaspar, L., 2013. Predicting runoff and sediment connectivity and soil erosion by water for different land use scenarios in the Spanish Pre-Pyrenees. Catena. doi:10.1016/j.catena.2011.01.001

Massa, F., 2000. Sédiments, physico-chimie du compartiment interstitiel et développement embryo-larvaire de la truite commune (Salmo trutta) : Etude en milieu naturel anthropisé et en conditions contrôlées. Institut national agronomique Paris-Grignon.

Matisoff, G., Wilson, C.G., Whiting, P.J., 2005. The7Be/210Pbxs ratio as an indicator of suspended sediment age or fraction new sediment in suspension. Earth Surf. Process. Landforms 30, 1191-1201. doi:10.1002/esp. 1270

Olley, J., Burton, J., Smolders, K., Pantus, F., Pietsch, T., 2013. The application of fallout radionuclides to determine the dominant erosion process in water supply catchments of subtropical South-east Queensland, Australia. Hydrol. Process. doi:10.1002/hyp.9422

Olley, J.M., Murray, a. S., Mackenzie, D.H., Edwards, K., 1993. Identifying sediment sources in a gullied catchment using natural and anthropogenic radioactivity. Water Resour. Res. 29, 1037.

Owens, P.N., Batalla, R.J., Collins, A.L., Gomez, B., Hicks, D.M., Horowitz, A.J., Kondolf, G.M., Marden, M., Page, M.J., Peacock, D.H., Petticrew, E.L., Salomons, W., Trustrum, N.A., 2005. Fine-grained sediment in river systems: environmental signifiance and management issues. River Res. Appl. 693-717.

Sabatier, P., Poulenard, J., Fanget, B., Reyss, J.-L., Develle, A.-L., Wilhelm, B., Ployon, E., Pignol, C., Naffrechoux, E., Dorioz, J.-M., Montuelle, B., Arnaud, F., 2014. Long-term relationships among pesticide applications, mobility, and soil erosion in a vineyard watershed. Proc. Natl. Acad. Sci. 111, 15647-15652. doi:10.1073/pnas.1411512111 
Shear, H., Watson, A.E.P., 1977. The fluvial transport of sediment associated nutrients and contaminants. Wind. Ontario Int. Jt. Comm.

Simonneau, A., Doyen, E., Chapron, E., Millet, L., Vannière, B., Di Giovanni, C., Bossard, N., Tachikawa, K., Bard, E., Albéric, P., Desmet, M., Roux, G., Lajeunesse, P., Berger, J.F., Arnaud, F., 2013. Holocene land-use evolution and associated soil erosion in the French Prealps inferred from Lake Paladru sediments and archaeological evidences. J. Archaeol. Sci. 40, 1636.

Sogon, S., 1999. Erosion des sols cultives et transport des matieres en suspension dans un bassin versant de brie : application des traceurs radioactifs naturels et mangnetiques. Paris 1.

Van Metre, P., Wilson, J., Fuller, C., Callender, E., Mahler, B., 2004. Collection, Analysis, and Age-Dating of Sediment Cores From 56 U.S. Lakes and Reservoirs Sampled by the U.S. Geological Survey, 19922001. U.S. Geol. Surv. Sci. Investig. Rep. 2004-5184.

Vandromme, R., Foucher, A., Cerdan, O., Salvador-Blanes, S., n.d. Quantification of bank erosion of artificial drainage networks using LIDAR data. Zeit. for Geomorph.

Vanmaercke, M., Poesen, J., Verstraeten, G., de Vente, J., Ocakoglu, F., 2011. Sediment yield in Europe: Spatial patterns and scale dependency. Geomorphology 130, 142.

Vidon, P., Cuadra, P.E., 2011. Phosphorus dynamics in tile-drain flow during storms in the US Midwest. Agric. Water Manag. doi:10.1016/j.agwat.2010.09.010

Walling, D.E., 1983. The sediment delivery problem. J. Hydrol. 65, 209-237. doi:http://dx.doi.org/10.1016/0022-1694(83)90217-2

Walling, D.E., Russell, M.A., Hodgkinson, R.A., Zhang, Y., 2002. Establishing sediment budgets for two small lowland agricultural catchments in the UK. Catena. doi:10.1016/S0341-8162(01)00187-4

Waters, T., 1995. Sediment in Streams Sources, Biological Effects and Control. American Fisheries Society Monograph 7. Bethesda, Maryland.

Wilkinson, B.H., 2005. Humans as geologic agents: A deep-time perspective. Geol. 33, 161-164. doi:10.1130/G21108.1 